

\title{
Phénotypes des allergies alimentaires : expériences des cohortes
}

\author{
Jocelyne Just, Flore Amat, A. Deschildre
}

\section{To cite this version:}

Jocelyne Just, Flore Amat, A. Deschildre. Phénotypes des allergies alimentaires : expériences des cohortes. Revue francaise d'allergologie, 2015, 55 (7), pp.474-479. 10.1016/j.reval.2015.08.002 . hal01206531

\section{HAL Id: hal-01206531 \\ https://hal.sorbonne-universite.fr/hal-01206531}

Submitted on 29 Sep 2015

HAL is a multi-disciplinary open access archive for the deposit and dissemination of scientific research documents, whether they are published or not. The documents may come from teaching and research institutions in France or abroad, or from public or private research centers.
L'archive ouverte pluridisciplinaire HAL, est destinée au dépôt et à la diffusion de documents scientifiques de niveau recherche, publiés ou non, émanant des établissements d'enseignement et de recherche français ou étrangers, des laboratoires publics ou privés. 
1 Phénotypes des Allergies alimentaires : Expériences des cohortes

2 Phenotype of food allergy: lessons from cohorts

3

4 Jocelyne JUST $^{1,2,}$ Flore AMAT ${ }^{1,2}$, Antoine DESCHILDRE ${ }^{3}$

5

6

1. Allergology Department, Centre de l'Asthme et des Allergies, Hôpital d'Enfants Armand-Trousseau (APHP)

2. Institut Pierre Louis d'Epidémiologie et de Santé Publique, Equipe EPAR, Sorbonne Universités, UPMC Univ Paris 06, UMR_S 1136, Paris, France

3. Pediatric Pulmonology and Allergy Department, Pôle enfant, Hôpital Jeanne de Flandre, CHRU de Lille and Université Nord de France, 59037 Lille, France

Adresse pour correspondance:

Pr. Jocelyne JUST

Centre de l'Asthme et des Allergies. Hôpital d'Enfants Armand-Trousseau (APHP) - 26, Avenue du Dr. Arnold Netter, 75571 PARIS Cedex 12. France.

Tel. +33144736317

Fax: +33144736635

E-mail: jocelyne.just@trs.aphp.fr

Mots clé : allergie alimentaire, cohorte

Key Word: food allergy, cohort 


\section{INTRODUCTION}

26

27

Les allergies alimentaires sont des maladies multiples en terme de sévérité, d'évolution, d'association potentielle à des comorbidités allergiques.

Dans la démarche d'une médecine personnalisée, l'approche phénotypique des maladies s'est développée au début du siècle, pour exemple, la prise en charge des maladies hématologiques, des cancers a évolué en fonction de biomarqueurs souvent d'origine génétique qui orientent de plus en plus les thérapeutiques ciblées.

Dans cette même démarche, l'asthme de l'enfant n'est plus considéré comme une maladie mais comme un syndrome relevant de phénotypes multiples.

Pour l'allergie alimentaire également, on peut penser que cette démarche pourrait aider le clinicien à mieux prendre en charge de façon personnalisée cette maladie aux visages multiples.

Les paramètres qui paraissent contributifs pour l'élaboration des phénotypes sont représentés par

- l'allergène (aliment et surtout ses déterminants épitopiques). Pour exemple chez l'enfant les allergènes alimentaires d'origine végétale ont plus tendance à perdurer que les allergies liées aux allergènes alimentaires d'origine animale comme le lait ou l'œuf ${ }^{1}$. Cependant, la multiplicité des reconnaissances épitopiques d'un même allergène, comme la caséine, est associée à la persistance de l'allergie au lait de vache ${ }^{2}$. De même la structure conformationnelle de l'aliment va modifier son allergénicité ${ }^{3}$. De cette façon, l'allergologie moléculaire nous a permis de mieux anticiper le risque des allergies alimentaires avec des allergènes potentiellement dangereux dans la famille des LTP (Lipid Transfer Protein) et des 
47 protéines de stockage ${ }^{4}$, responsables plus volontiers d'anaphylaxie alimentaire et d'allergie,

48 les syndromes oraux retrouvés avec la famille des PR10 dans le syndrome pollens-fruits. -les seuils réactogènes variables en fonction de l'individu mais aussi de conditions de vie comme association à l'effort dont le premier exemple est l'anaphylaxie d'effort au blé 5 .

- l'association à des comorbidités allergiques comme l'asthme et la dermatite atopique ou encore la multiplicité des allergies alimentaires qui va aggraver le pronostic de chacune d'entre elles.

$\mathrm{Au}$ cours des chapitres qui vont suivre, nous allons donc aborder différents phénotypes d'allergie alimentaire à partir de cohortes néonatales et de cohortes de patients atteints d'allergie alimentaire, évaluées par des analyses en cluster, en s'orientant sur les allergies alimentaires les plus préoccupantes, c'est à dire 1) les allergies alimentaires à risque de passage d'une maladie atopique à l'autre au cours de l'enfance (de l'allergie alimentaire et/ou à l'allergie cutanée à l'allergie respiratoire) 2) puis les allergies alimentaires sévères.

\section{Phénotypes à risque de passage d'une maladie atopique à une autre}

Les mécanismes et les facteurs de risque de sensibilisation allergénique alimentaire sont différents suivant le profil de sensibilisation allergénique

Une étude australienne de cohorte de 5000 nouveau-nés issus de la population générale et suivis de façon prospective essaie de répondre à cette question ${ }^{6}$. A l'âge de 12 mois, une analyse statistique non supervisée de type LCA (Latent Class Analysis) est réalisée en fonction des paramètres suivants: (1) sensibilisations allergéniques détectées par les tests cutanés allergologiques, (2) résultats des tests de provocation allergénique à l'œuf, à l'arachide, au sésame, (3) eczéma à début précoce ou retardé (avant ou après l'âge de 4 mois), (4) sifflements dans la première année de vie. Cinq phénotypes sont ainsi identifiés : absence 
de maladie allergique (70 \% des cas), un phénotype avec eczéma sans sensibilisation allergénique (16\% des cas), une sensibilisation isolée à l'œuf (9\% des cas), des allergies multiples notamment à l'arachide (3\% des cas) et des allergies multiples notamment à l'œuf ( $2 \%$ des cas). En comparaison au groupe sans allergie, les groupes allergiques avaient les mêmes facteurs de risque, c'est-à-dire (1) être issu de famille d'immigrée particulièrement d'Asie, (2) avoir une introduction retardée de l'œuf, (3) être plutôt de sexe masculin, (3) avoir des antécédents familiaux d'allergie (4) et enfin pour tous les 5 phénotypes, avoir des animaux dans les premières années de vie étaient protecteurs de ces allergies alimentaires.

Alors que des facteurs tels que la présence d'une mutation de la filaggrine, le taux de vitamine $\mathrm{D}$ et la présence d'une fratrie différaient d'un phénotype à l'autre. Ainsi les mutations de la filaggrine étaient surtout retrouvés pour le phénotype avec eczéma sans sensibilisation allergénique [aOR 2,37 (95\% intervalle de confiance 1,27-4,43, p=0,01)] et dans le phénotype d'allergies multiples notamment à l'œuf [aOR 1,78 (95\% intervalle de confiance $0,99-3,19, \mathrm{p}=0.05)]$.

La sensibilisation multiple aux trophallergènes est à risque de sensibilisation aux allergènes inhalés: cohorte ORCA (Observatory of Respiratory risks linked with Cutaneous Atopy) ${ }^{7}$ 229 enfants ont été inclus et suivis dans cette cohorte d'enfants atteints de dermatite atopique modérée à sévère, à début précoce. Dans cette étude la sensibilisation aux trophallergènes diminue de $58 \%$ avant l'âge de 1 an à $34 \%$ à l'âge de 5 ans alors que la sensibilisation aux allergènes inhalés passe de $17 \%$ avant l'âge de 1 an à $67 \%$ à l'âge de 5 ans.

Une analyse multivariée montre que c'est la sensibilisation multiple aux trophallergènes qui est le meilleur facteur prédictif du risque de sensibilisation aux allergènes inhalés à l'âge de 6 ans avec un odd ratio de 3,72 (intervalle de confiance 1,68-8,30; $\mathrm{p}<0,001$ ) (Figure $\mathrm{n}^{\circ} 1$ ). 
D'autres études, comme la cohorte MAAS (The National Asthma Campaign Manchester Asthma and Allergy Study) réalisée en population générale, montrent que la sensibilisation allergénique multiple et précoce va prédisposer à l'asthme persistant, exacerbateur au cours de l'enfance ${ }^{8}$. Ce dernier résultat a été validé par Lazic et al. ${ }^{9}$, qui montre que les phénotypes allergiques ne changent pas au cours du temps et que le phénotype allergique associé à des sensibilisations allergéniques multiples est à risque d'asthme persistant au cours de l'enfance.

\section{La sensibilisation allergénique multiple aux trophallergènes est à risque d'apparition} d'asthme et de dermatite atopique au cours de l'enfance: Etude WHEAL (Wayne County Health Environment, Allergy and Asthma Longitudinal Study) $)^{10}$

Dans une cohorte néonatale de 594 enfants issus de la population générale, des sensibilisations allergéniques sont recherchées à l'âge de 2 ans envers 10 allergènes inhalés ou ingérés différents (acariens, chat, chien, pollens de graminées, arachide, Alternaria, œuf, arachide, lait blatte). L'atopie est définie comme au moins une IgE supérieure à $0,35 \mathrm{IU} / \mathrm{ml}$.

Quatre classes sont retrouvées par une analyse statistique non supervisée, une classe sans sensibilisation (76 \% de la population), une classe hautement sensibilisée $(2,7 \%$ de la population), une classe avec sensibilisation au lait et à l'œuf (15,3\% de la population) et une classe avec une sensibilisation à l'arachide isolée (5\% de la population). La classe ayant une sensibilisation élevée était le plus à risque d'avoir un asthme à l'âge de 4 ans (odds ratio 5,3, intervalle de confiance 1,6 - 17,4), alors que la sensibilisation au lait et l'œuf et à l'arachide isolée ne l'était pas.

La sensibilisation allergénique multiple aux trophallergènes est à risque de passage de la dermatite atopique à l'asthme : cohorte ORCA 
De nombreuses études concluent que la marche atopique doit être revisitée car elle ne représente pas une évolution fréquente de la «carrière allergique ».

122 Dans la cohorte ORCA, une étude en cluster utilisant 9 variables pertinentes chez 214 enfants

123 ayant un eczéma à début précoce modéré à sévère a été réalisé avec une évaluation du risque d'évolution vers l'asthme à l'âge de 6 ans ${ }^{11}$.

Trois phénotypes de dermatite atopique à début précoce ont pu être retrouvés, un phénotype de dermatite atopique légère en intensité (évaluée par le SCORAD) nommé «dermatite atopique légère sans sensibilisation allergénique », un phénotype caractérisé par un SCORAD élevé et des sensibilisations aux trophallergènes dans $99 \%$ des cas (multiples dans 96,4 \% des cas) et aux pneumallergènes dans plus de $26 \%$ des cas, que nous avons dénommé « dermatite atopique sévère avec sensibilisations multiples » et enfin un troisième phénotype avec une histoire parentale d'asthme, une dermatite atopique modérée et une sensibilisation le plus souvent unique aux trophallergènes, dénommé «dermatite atopique avec histoire familiale d'asthme ». Le risque d'évoluer vers l'asthme à l'âge de 6 ans est surtout élevé dans les deux derniers phénotypes, c'est-à-dire en cas de sensibilisations allergéniques multiples ou en cas de phénotype associé à des antécédents familiaux d'asthme.

Dans cette étude une fois de plus, la multi-sensibilisation précoce avant l'âge de 1 an, notamment aux trophallergènes, va prédisposer à une dermatite atopique plus sévère et à un risque de passage à l'asthme et donc caractériser ce phénotype rare et sévère.

Ce résultat est en accord avec l'étude de Belgrave et al $^{12}$ qui montre qu'en utilisant une analyse statistique non supervisée dans une cohorte néonatale suivie de façon prospective (MAAS), la classique «marche atopique » ne concernait que $3 \%$ de la population générale. C'est dans ce dernier phénotype que la sensibilisation allergénique était la plus fréquente avec un odd ratio de 32,6 (intervalle de confiance 23,6-45,2; $\mathrm{p}<0.001$ ). 
147 Dès le plus jeune âge, chez les enfants d'âge préscolaire, l'association asthme et allergie 148 alimentaire prédispose à la persistance de l'asthme allergique au cours de l'enfance. 149 Cependant il importe de définir quels phénotypes d'allergie alimentaire sont le plus à risque 150 de passage de l'allergie alimentaire à l'asthme.

Dans la première cohorte de TAP réalisée chez 551 nourrissons présentant un asthme à début précoce, 3 phénotypes d'asthme ont pu être mis en évidence dont un dénommé «asthme

153 atopique à facteurs déclenchants multiples » dans lequel une allergie alimentaire est retrouvée dans $36 \%$ des cas, en association à une dermatite atopique dans $75 \%$ des cas $\left(\text { Figure } \mathrm{n}^{\circ} 2\right)^{13}$.

Ce phénotype d'asthme à début précoce et allergique était à fort risque d'évolution vers un asthme allergique comme en témoigne le suivi de ces nourrissons asthmatiques. En effet, dans ce phénotype d'asthme allergique à facteurs déclenchant multiples, il n'y avait aucune rémission d'asthme à l'âge de 5 ans (Figure $\left.n^{\circ} 3\right)^{14}$. Ce phénotype d'asthme à facteurs déclenchant multiples persistait avec un asthme modéré à sévère dans les 2 tiers des cas à l'âge de 5 ans.

Chez le jeune enfant, la rhinite allergique est une maladie rare et sous diagnostiquée. Dans une étude réalisée chez 127 nourrissons atteint d' asthme à début précoce et participant au TAP, les déterminants de la rhinite allergique ont été recherchés en comparant un groupe de jeunes enfants atteint d'asthme à début précoce associé à une rhinite allergique et un groupe de jeunes enfants atteint d'asthme sans rhinite allergique. En analyse multi variée, les déterminants de la rhinite allergique sont principalement la sensibilisation à l'arachide oddratio de $6,75(\mathrm{p}=0,02)$, ''allergie alimentaire oddratio 4,31 $(\mathrm{p}=0,026)$, l'exposition aux moisissures oddratio à $3,80(\mathrm{p}=0,01)$ et l'histoire parentale de rhinite allergique oddratio 1,42 
$(\mathrm{p}=0,46)$. Il y a donc une forte association entre la rhinite allergique et l'allergie alimentaire notamment à l'arachide ce qui pose le problème d'un phénotype particulier associant

172 l'allergie alimentaire, la rhinite allergique et l'asthme à début précoce, à fort risque de persistance au cours de l'enfance comme précédemment évoqué dans d'autres cohortes de 174 TAP.

\section{Les phénotypes des allergies alimentaires sévères}

177 Le poids des comorbidités et du genre dans la sévérité de l'allergie à l'arachide : Cohorte

\section{MIRABEL}

La cohorte MIRABEL est une cohorte majoritairement réalisée en France, de 704 sujets allergique à l'arachide (principalement des enfants) ayant eu pour 247 d'entre eux un test de provocation (TPO) à l'arachide. Des analyses statistiques non supervisées réalisées dans cette population montrent 3 phénotypes indépendants : un premier phénotype avec une allergie sévère à l'arachide comme en témoigne un très haut pourcentage de TPO positifs ( $92 \%)$, avec un seuil réactogène bas et peu de comorbidité allergiques $(2 \%$ de syndrome dermorespiratoire, aucun cas d'asthme isolé, de dermatite atopique isolée ou d'allergie alimentaire multiple); un deuxième phénotype avec des comorbidités allergiques multiples (100\% de syndrome dermo-respiratoire) et une allergie sévère à l'arachide avec la dose médiane cumulée réactogène la plus basse par rapport aux 2 autres clusters (112mg d'équivalent protéique d'arachide ); enfin un $3^{\text {ème }}$ phénotype ayant une allergie légère avec un fort pourcentage de sujets «sensibilisés », c'est-à-dire ne réagissant pas à une dose médiane cumulée de 770mg de protéines d'arachide.

Par ailleurs une plus grande fréquence des deux phénotypes d'allergie à l'arachide sévère est retrouvée dans cette étude chez les filles. 
Cette étude renforce le rôle du poids des comorbidités (notamment l'association avec l'asthme et la dermatite atopique) sur la sévérité de l'allergie alimentaire et souligne la prédominance féminine de ces allergies sévères.

\section{Allergie alimentaire sévère et allergologie moléculaire, analyses en cluster}

Très récemment publiée dans Allergy ${ }^{15}$, une étude a été réalisée dans une large population de patients souffrant d'allergie alimentaire en Italie. L'objectif était de corréler le profil des sensibilisations moléculaires allergéniques par des analyses en cluster et les phénotypes cliniques d'allergie alimentaire. Quatre-vingt-deux pour cent des sujets (94\% si on comptait les moins de 6 ans) avaient une LTP positive pour Pru p3 et $71 \%$ pour Jug r3. Les participants qui avaient plus de 5 LTP positives reportaient le plus haut risque de réaction systémique. Seules Art v3 et Pla a3 (en rapport avec des LTP d'armoise et de pollens d'arbre respectivement) étaient associés à des symptômes respiratoires. La co-sensibilisation à Parj2 (LTP de la pariétaire) et PR-10 ou profiline était associée à un moindre risque de réaction allergique sévère. En conclusion, les auteurs soulignaient que le type de sensibilisation allergénique aux LTP est important pour la présentation clinique et l'identification de facteurs de risque de forme sévère.

Récemment, Martinez-Botas et $\mathrm{al}^{16}$. ont eu pour objectif de prédire l'efficacité d'une immunothérapie orale (ITO) au lait par des biomarqueurs, en l'occurrence le profil de réponse épitopique IgE et IgG4 induit par l'ITO. L'étude porte sur 25 enfants allergiques au lait traités par ITO et 7 enfants allergiques au lait non traités par ITO (groupe contrôle). Le groupe traité est divisé en 3 groupes à risque faible, modéré et élevé de risque de réactions syndromiques au cours de l'ITO (plus de 7 réactions) et également en fonction du temps nécessaire à terminer l'ITO (c'est-à-dire ingérer sans problème au moins $200 \mathrm{ml}$ de lait). Les sujets à haut risque ont un nombre statistiquement plus important d'IgE dirigés contre des 
219 peptides de la caséine (et à moindre degré d'IgG4). L'analyse en cluster du groupe à risque 220 élevé identifie 13 régions de la caséine de la réponse $\operatorname{IgE}$ qui différent significativement par 221 rapport aux autres groupes. Les analyses bioinformatiques permettent de sélectionner 2 séries

222 de 16 IgE dirigés contre des peptides de la caséine (avant le démarrage de l'ITO) qui 223 prédisent la tolérance $(\mathrm{R} 2=0.858)$ et l'efficacité $(\mathrm{R} 2=0.732)$, de l'ITO.

\section{Conclusions}

226 L'ensemble de ces résultats supportent l'hypothèse que l'expression clinique des allergies 227 alimentaires notamment en termes de sévérité des phénotypes et surtout des endotypes 228 (attachés à des biomarqueurs) est surtout représentée par la spécificité «moléculaire » des $\operatorname{IgE}$ 229 mais aussi leur multiplicité et leur précocité d'apparition au cours de l'enfance.

\section{Déclaration d'intérêts}

232 Les auteurs ne déclarent aucun conflit d'intérêt en rapport avec cet article 
(a) 507

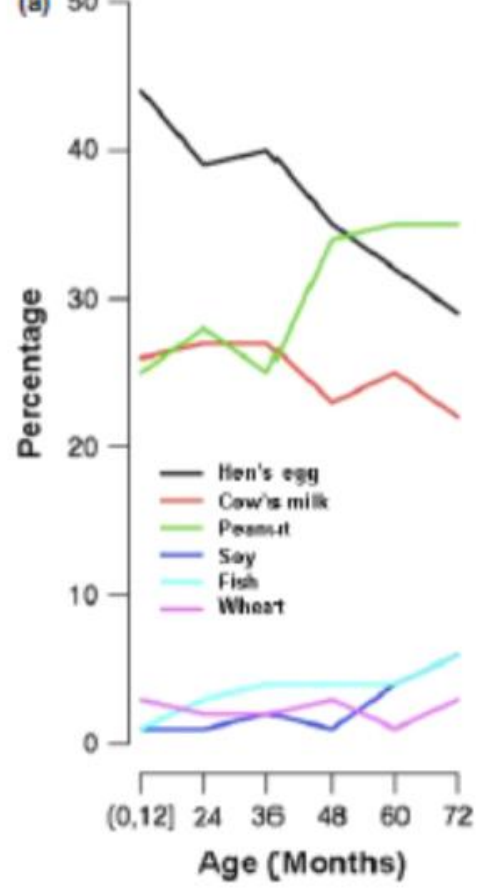

(b) $50-1$ -



Figure $n^{\circ}$ 1: évolution des sensibilisations allergéniques dans une cohorte de dermatite 

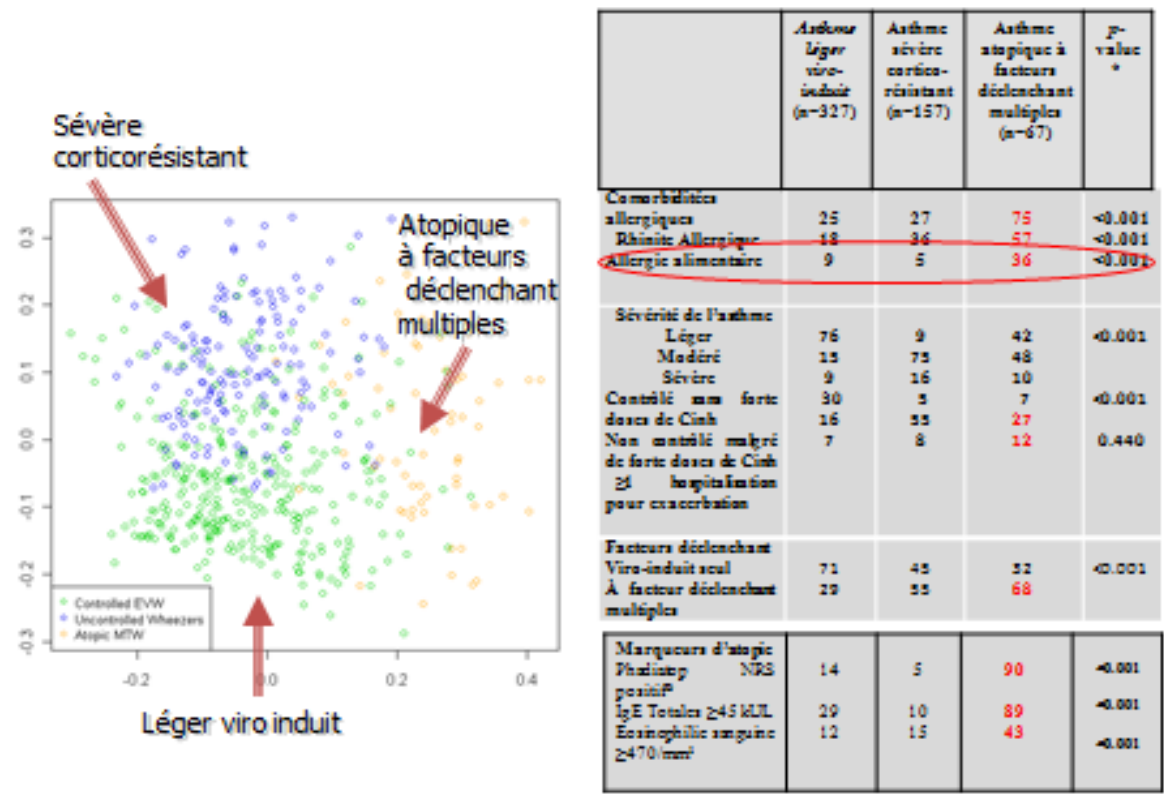

Figure $n^{\circ} 2$ Phénotypes d'asthme à début précoce du nourrisson selon référence $n^{\circ} 13$ 


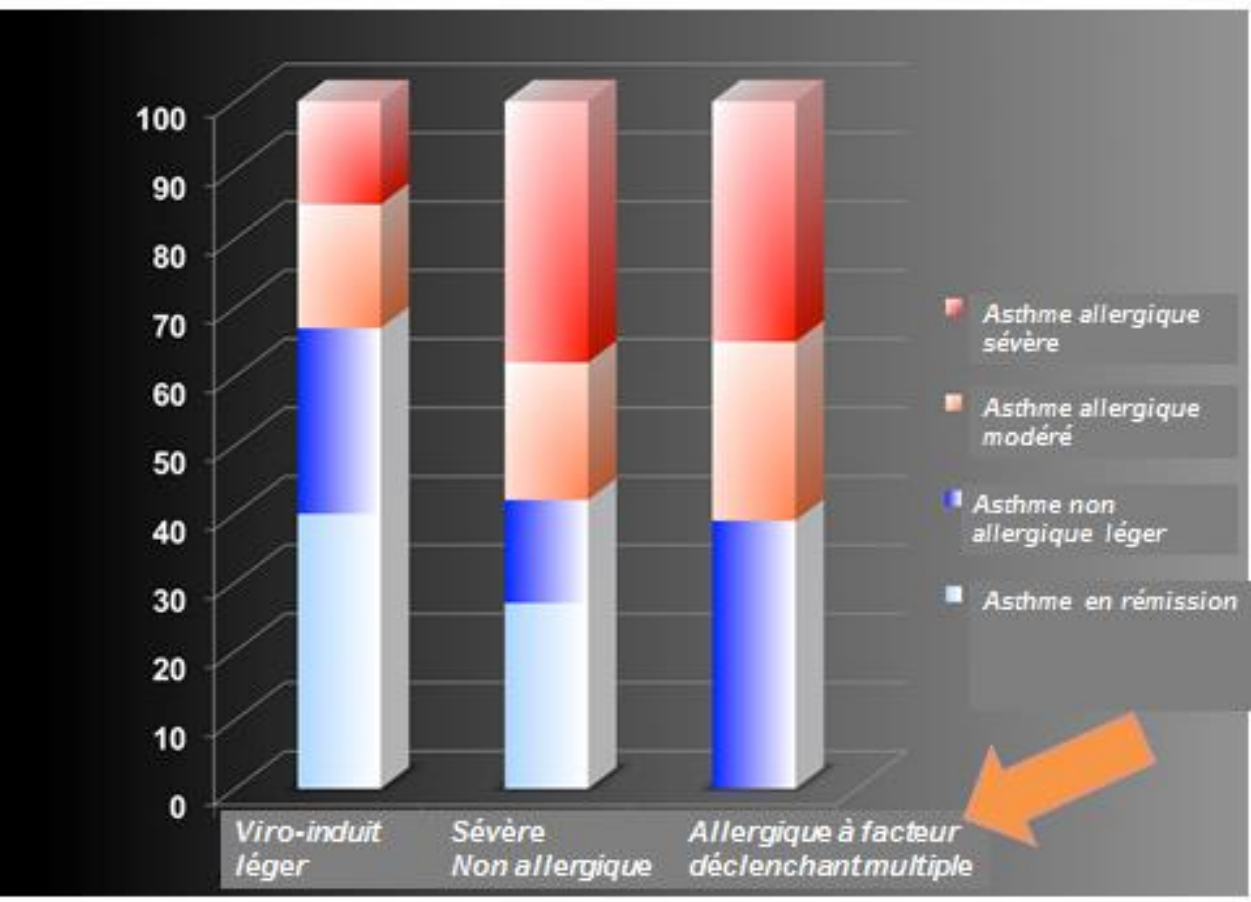

248

2.

249 3. Figure $n^{\circ} \mathbf{3}$ Devenir de l'asthme précoce au cours de l'enfance selon la référence $\mathbf{n}^{\circ} 14$

250 


\section{Références}

${ }^{1}$ Savage J, Johns CB. Food allergy: epidemiology and natural history. Immunol Allergy Clin North Am. 2015;35(1):45-59.

${ }^{2}$ Lemon-Mulé H, Sampson HA, Sicherer SH, Shreffler WG, Noone S, Nowak-Wegrzyn A. Immunologic changes in children with egg allergy ingesting extensively heated egg. J Allergy Clin Immunol. 2008;122(5):977-983.

${ }^{3}$ Cerecedo I, Zamora J, Shreffler WG, Lin J, Bardina L, Dieguez MC, Wang J, Muriel A, de la Hoz B, Sampson HA. Mapping of the IgE and IgG4 sequential epitopes of milk allergens with a peptide microarray-based immunoassay.J Allergy Clin Immunol. 2008 Sep;122(3):58994.

${ }^{4}$ Astier C, Morisset M, Roitel O, Codreanu F, Jacquenet S, Franck P, and al. .Predictive value of skin prick tests using recombinant allergens for diagnosis of peanut allergy.J Allergy Clin Immunol. 2006 ;118(1):250-6.

${ }^{5}$ Pastorello EA, Farioli L, Stafylaraki C, Scibilia J, Mirone C, and al. Wheat-dependent exercise-induced anaphylaxis caused by a lipid transfer protein and not by $\omega-5$ gliadin. Ann Allergy Asthma Immunol. 2014;112(4):386-7.

${ }^{6}$ Peters RL, Allen KJ, Dharmage SC, Lodge CJ, Koplin JJ, et al. Differential factors associated with challenge-proven food allergy phenotypes in a population cohort of infants: a latent class analysis. Clin Exp Allergy. 2015;45(5):953-63.

7 Just J, Deslandes-Boutmy E, Amat F, Desseaux K, Nemni A, Bourrat E, et al. Natural history of allergic sensitization in infants with early-onset atopic dermatitis: results from ORCA Study. Pediatr Allergy Immunol. 2014 ;25(7):668-73.

${ }^{8}$ Simpson A, Tan VYF, Winn J, Svensén M, Bishop CM, Heckerman DE, et al. Beyond atopy: multiple patterns of sensitization in relation to asthma in a birth cohort study. Am $\mathrm{J}$ Respir Crit Care Med 2010; 181:1200-1206.

${ }^{9}$ Lazic N, Roberts G, Custovic A, Belgrave D, Bishop CM, Winn J, et al. Multiple atopy phenotypes and their associations with asthma: similar findings from two birth cohorts. Allergy. 2013; 68: 764-70.

${ }^{10}$ Havstad S, Johnson CC, Kim H, Levin AM, Zoratti EM, Joseph CL et al. Atopic phenotypes identified with latent class analyses at age 2 years. J Allergy Clin Immunol. 2014;134(3):722-727.

${ }^{11}$ Amat F, Saint-Pierre P, Bourrat E, Nemni A, Couderc R, Boutmy-Deslandes Eet al. EarlyOnset Atopic Dermatitis in Children: Which Are the Phenotypes at Risk of Asthma? Results from the ORCA Cohort. PLoS One. 2015;10(6):1-14. 
${ }^{12}$ Belgrave DC, Granell R, Simpson A, Guiver J, Bishop C, Buchan I, et al. Developmental profiles of eczema, wheeze, and rhinitis: two population-based birth cohort studies. PLoS Med. 2014; 11(10): 1-14.

${ }^{13}$ Just J, Gouvis-Echraghi R, Couderc R, Guillemot-Lambert N, Saint-Pierre P. Two novel severe wheezy young children phenotypes: boys atopic multiple-trigger and girls non-atopic uncontrolled wheeze. J Allergy Clin Immunol 2012; 130: 103-110.

${ }^{14}$ Just J, Saint-Pierre P, Gouvis-Echraghi R, Boutin B, Panayotopoulos V, Chebahi N, et al. Wheeze phenotypes in young children have different courses during the preschool period. Ann Allergy Asthma Immunol. 2013; 111: 256-261.

${ }^{15}$ Scala E, Till SJ, Asero R, Abeni D, Guerra EC, Pirrotta L, Pet al. Lipid transfer protein sensitization: reactivity profiles and clinical risk assessment in an Italian cohort. Allergy April 2015

${ }^{16}$ Martínez-Botas J, Rodríguez-Álvarez M, Cerecedo I, Vlaicu C, Diéguez MC, GómezCoronado D, et al. Identification of novel peptide biomarkers to predict safety and efficacy of cow's milk oral immunotherapy by peptide microarray. Clin Exp Allergy. 2015;45(6):107184. 